\title{
Injections of concentrated bone marrow aspirate as treatment for Discogenic pain: a retrospective analysis
}

\author{
Michael Wolff ${ }^{*} \mathbb{D}$, Jon Mark Shillington ${ }^{1}$, Christopher Rathbone², Shawn K. Piasecki ${ }^{3}$ and Brian Barnes ${ }^{3}$
}

\begin{abstract}
Background: There are an overwhelming number of patients suffering from low back pain (LBP) resulting from disc pathology. Although several strategies are being developed pre-clinically, simple strategies to treat the large number of patients currently affected is still needed. One option is to use concentrated bone marrow aspirate (cBMA), which may be effective due to its intrinsic stem cells and growth factors.
\end{abstract}

Methods: Thirty-three patients who received intradiscal injections of cBMA to relieve LBP were followed up based on Numeric Rating Scale (NRS), Oswestry Low Back Pain Disability Index (ODI), and Short Form-36 Health Survey (SF-36) scores. Patients were also subdivided into those with a pre-injection NRS $>5$ and pre-injection NRS $\leq 5$. The proportion of patients demonstrating at least 50\% improvement (and 95\% confidence intervals) from baseline at five follow-up visits for each outcome was evaluated.

Results: At least 50\% improvement in NRS was observed for 13.8, 45.8, 41.1, 23.5, and 38.9\% of patients across five follow-up visits, out to 1 year. When stratified by high $(>5)$ versus low $(\leq 5)$ baseline NRS scores, the values were $14.3,45.5,71.4,22.2$, and $44.4 \%$ among those with high baseline pain, and 13.3, 46.2, 20.0, 25.0, and 33.3\% among those with low baseline pain. The 50\% improvement rates across visits were 4.3, 28.6, 30.0, 22.2, and 30.8\% for SF36 , and $4.2,26.7,36.4,55.6$, and $30.8 \%$ for ODI.

Conclusions: Intradiscal CBMA injections may be effective to reduce pain and improve function. Patients with relatively higher initial pain may have potential for greatest improvement.

Keywords: Intradiscal, Low back pain, Platelet-rich plasma, Bone marrow aspirate, Discogenic, Mesenchymal stem cell

\section{Background}

Low back pain (LBP) is among the most prevalent and costly of musculoskeletal disorders. Greater than $80 \%$ of the U.S. population has at least one episode of LBP and the associated costs due to medical expenses and lost wages can exceed $\$ 100$ billion annually $[1,2]$. LBP is commonly pain resulting from pathological changes to the intervertebral disc (IVD) with discogenic pain being one of the main contributors to LBP [3]. The identification of non-surgical therapies with potential to treat discogenic pain at the level of the intervertebral disc could

\footnotetext{
* Correspondence: mww@swssaz.com

'Southwest Spine and Sports, 9913 N. 95th St, Scottsdale, AZ 85258, USA Full list of author information is available at the end of the article
}

dramatically improve patient quality of life and relieve a large national financial burden.

The IVD is comprised of an outer annular fibrosis (AF) and inner nucleus pulposus (NP). The AF is composed of concentric, dense lamellae of highly extracellular matrix-oriented type I \& II collagen fibers while the $\mathrm{NP}$ is a less structured gelatinous extracellular matrix rich in proteoglycans (including aggrecans) and type II collagen [4-6]. Disc degeneration that occurs over time and/or subsequent to trauma is not surprising given the limited vascularity supplying the AF and NP [7]. Accordingly, a diminished capacity to cope with an inflammatory environment results in impaired IVD cell function, a situation that ultimately manifests as decreased proteoglycan synthesis and nuclear dehydration $[4,7,8]$. The 
untoward consequence of these events may be the development of local annular fissures, delamination, eventual internal annular disc disruption, and/or disc space narrowing. These factors may contribute to the development of low back pain that is discogenic in etiology based on a combination of the mechanical and biochemical changes within the disc. Therapies with the potential to shift the balance to a healthier IVD environment may have the ability to decrease discogenic pain $[7,9,10]$.

The use of point-of-care autologous therapies, namely platelet-rich plasma (PRP) and bone marrow aspirate (BMA)-derived therapies (commonly referred to as bone marrow aspirate concentrate (BMAC), bone marrow concentrate (BMC), or concentrated bone marrow aspirate (cBMA)) have been demonstrated to be a means to decrease pain for a wide variety of orthopedic applications [11-17]. These and other preliminary cBMA studies are extremely valuable tools for the modern clinician, yet the issue of which applications are most appropriate for utilizing this technology remains. This is most likely because researchers are still uncertain of the exact detailed mechanism by which cBMA acts [18]. Many hypotheses exist, including that the results may be due to increased anti-inflammatory and anabolic cytokine and growth factor signaling contained within the physiologically buffered concentrate of BMA $[18,19]$. Another theory is that the Mesenchymal Stem Cells (MSCs) found in cBMA [20] may directly mediate tissue repair, act via a paracrine pathway, or both $[18,21]$. It is entirely plausible that cBMA acts by a combination of the hypothesized mechanisms mentioned, yet future studies are necessary for characterization in further detail. While continued insight into the mechanism of action is in need of further elucidation, one concern that most are in agreement with today is that injections of PRP and cBMA appear to be at least safe and reasonably effective as an interventional therapy for patients suffering from a range of orthopedic conditions [11-17, 22].

The preliminary PRP and cBMA injection studies have demonstrated promising potential to alleviating discogenic pain as a safe alternative for LBP relief [23-25]. For example, Pettine et al. reported favorable results for the use of cBMA when they demonstrated significant, prolonged reductions in pain after intradiscal injections [23, 26, 27]. Preclinical work in a chimeric rodent rotator cuff model by Nakagawa et al. suggests that these bone marrow-derived cells, in the presence of a fibrocartilage layer, have the potential to differentiate into chondrogenic cells and proliferate when transplanted in vivo [28]. Although promising, the results have yet to be confirmed in an IVD model. Nonetheless, the potential for the application of autologous therapies as a source of regenerative factors for healing within the virtually avascular IVD may be an elegant solution to an otherwise complex condition. Herein, our retrospective analysis of 33 patients adds further support for the concept of utilizing point-of-care autologous therapies to treat discogenic low back pain.

\section{Methods}

\section{Patient selection for CBMA treatment}

Each patient displayed objective findings of discogenic degenerative changes via MRI evidence which included disc desiccation, disc bulge or small contained protrusion (>6 mm), and/or posterior annular tear (high intensity zone, HIZ). Patients selected as candidates for treatment also had $\geq 50 \%$ disc height retained compared to other lumbar discs that were considered normal. The presence of Modic changes were not considered within our patient selection. By history, patients had subjective findings consistent with chronic discogenic LBP. Pain was reported with positions and/or activities such as sitting, bending, lifting and/or transitioning from sit to stand, or maintaining a forward flexed position at the waist. Patients reported that LBP was greater than radicular symptoms (if they had any). Physical exam findings included: reports of low back pain with range of motion (forward flexion > extension), normal neurological exam (i.e., negative for weakness, sensory loss, Muscle Stretch Reflex abnormality, and/or negative SLR).

Provocative discography was performed on all patients to confirm discogenic pain and internal disc disruption. All discograms were performed by a single physician (MWW), by SIS standard guidelines [29] using a pressure manometer device and a two-needle technique with a 22-guage needle for disc entry. All patients had at least one control (i.e., normal pressure, painless) disc. Pressurization of the disc was limited to $50 \mathrm{psi}$ above opening pressure or a volume of $3 \mathrm{ml}$ (whichever came first). Concordant pain response was $\geq 6$ (out of 10), described as typical pain by the patient. Volume injected into the disc, opening pressure (pressure at which contrast is seen in the disc), provocation pressure (pressure when pain is first reported), peak pressure (final pressure), character of pain response (concordant, discordant, partial concordant, no pain) was recorded for all patients. Patients were subsequently sent to obtain a post-discogram CT scan. The Modified Dallas discogram scale was used for interpretation of disc disruption on all post discogram CT scans obtained. Patients with positive concordant or partial concordant pain on discography and anatomical evidence of internal disc disruption on post-discography CT scan (Grades 1-4) were selected for treatment. No Grade 5 tears or full thickness tears were treated. All intradiscal cBMA injections were performed on a later date than the time of discography. 
Study procedures were approved by the Honor Health Institutional Review Board (Scottsdale, AZ). Patients were identified by retrospective review of medical records between April 2010 and April 2015 and included those with chronic low back pain, confirmed to be discogenic in etiology. All patients had a long history of chronic LBP, typically greater than 1 year. All patients had a history of extensive conservative therapy without significant long-term benefit prior to intradiscal cBMA treatment including use of medications (NSAIDs and/or narcotics), physical therapy, and injections (epidural and/or medial branch blocks that were negative). A total of 33 patients who were treated during this time frame were reviewed (14 female, 19 male) (Table 1). During the period of observation after treatment, patients were scheduled for standard follow up appointments at 2 weeks, and 2, 3, 6, 12 months for a total of 5 visits. They were given post-injection pain medication if mutually agreed upon including NSAIDs and/or narcotics that were taken on an as-needed basis. No patients underwent other spinal interventions, such as epidurals, additional intradiscal therapies, or spinal fusion/surgery for the length of observation. There were no remarkable adverse events or complications to report.

\section{Bone marrow collection and processing}

All procedures were performed in an ambulatory surgery center. Standard sterile technique and fluoroscopic guidance was used per protocol. Minimal to moderate conscious sedation (Versed, Fentanyl) was used as necessary to ensure patient comfort during the procedure. Local anesthesia using $2 \%$ lidocaine was used superficially and to the level of the periosteum overlying the Posterior

Table 1 Candidate Selection Criteria for cBMA Treatment

Inclusion Criteria ( $n=33)$
- Refractory LBP displaying discogenic degenerative changes via MRI
- Positive concordant or partial concordant pain on discography and
demonstrated internal disc disruption via CT scan (1,2, or 3-level disc
pathology accepted)
- $\geq 50 \%$ disc height maintained at level(s) of treatment
- No responsiveness to conservative therapy
- Subjective findings of chronic LBP suggestive of discogenic etiology
- Patients treated between Apr 2010 - Apr 2015
- Patients desiring to be treated with concentrated autologous bone
marrow aspirate at the level(s) of treatment
Exclusion Criteria
- LBP caused by any other etiologies (facet pain, stenosis, etc.)
- <50\% disc height maintained at level(s) of treatment
- Full-thickness tears
- Patients who underwent any additional therapies during the follow
up period

Superior Iliac Spine (PSIS). This was completed using a 27-gauge 1.5-in. and/or 25-gauge 3.5-in. needle as necessary. An 11-gauge Jamshidi bone marrow aspiration needle was advanced through the anesthetized tissue under fluoroscopic guidance until contact was made with the periosteum of the PSIS. The needle was advanced through the cortex of the PSIS and into the trabecular bone. Similar to previously described "aspirate-rotate-aspirate" protocols [30, 31], $52 \mathrm{ml}$ of BMA was drawn into a 60-ml syringe containing $8 \mathrm{ml}$ anti-coagulant citrate dextrose solution (ACD-A, Isto Biologics, Hopkinton, MA) rotating the needle in $5 \mathrm{ml}$ aliquots at each level, and slightly withdrawing every $20 \mathrm{ml}$. If more than one disc level was being treated then the same procedure was repeated on the contralateral side. Thereafter, $60 \mathrm{ml}$ of anticoagulated BMA was placed in the Magellan Autologous Platelet Separator System (Isto Biologics, Hopkinton, MA) and spun using its Standard Cycle (2800 $\mathrm{rpm}$ for $\sim 8 \mathrm{~min}$ followed by $3800 \mathrm{rpm}$ for $\sim 8 \mathrm{~min}$ ) to obtain 3 to $6 \mathrm{ml}$ of cBMA, containing platelets, regenerative cells including mesenchymal stem cells (MSCs), and growth factors.

\section{Intradiscal injections}

Each target disc was identified under fluoroscopy using T12 as a reference. Superficial tissue was anesthetized to the level of the superior articular process with 1-2 $\mathrm{ml}$ of $1 \%$ preservative-free lidocaine. A standard, two-needle technique using an 18-gauge skin needle and a 22-gauge intradiscal needle was fluoroscopically guided in a right or left extra-pedicular approach to place the needle tip into the nucleus of the disc. Proper placement was confirmed in two planes (Anterior/Posterior and Lateral) and pictures of final placement were printed. A predetermined volume of cBMA (3 ml or less) was injected intradiscally. After cBMA injection, needles were removed carefully, and bandages and pressure were applied to the injection site(s) under sterile conditions.

\section{Outcome measures}

Patients underwent an examination including the Oswestry Low Back Pain Disability Index (ODI) Questionnaire, Short Form-36 (SF-36) Health Survey, and Numeric Rating Scale (NRS) assessments pre-injection, and at $2,6-8,12,24$, and $\geq 52$ weeks post-treatment.

\section{Statistical analysis}

SAS Version 9.4 (SAS Institute, Cary, NC) was used for all analyses. The effects of cBMA on NRS, SF-36, and ODI measures were evaluated using categorical analysis to examine the proportion of patients experiencing $\geq 50 \%$ change (and 95\% confidence intervals) in NRS, SF36 , and ODI over time in accordance with baseline pain levels (Fig. 1). The proportion of patients experiencing 

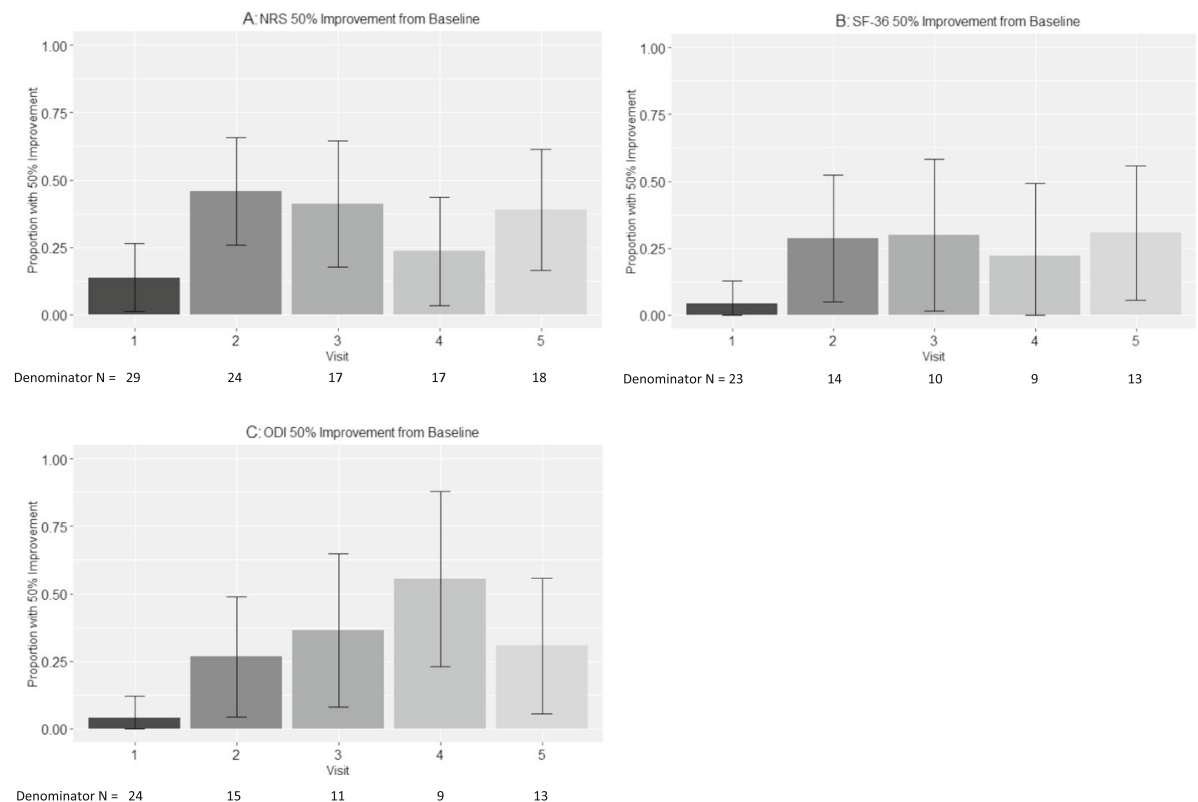

Fig. 1 Proportion of patients with Numeric Rating Scale (NRS; a), Short Form-36 Health Survey (SF-36; b) and Oswestry Low Back Pain Disability Index (ODl; c) score improvements of at least 50\%, and the number of patients included at each time point. Values are proportions and $95 \%$ confidence intervals. Visits are defined as follows: visit 1 ( 2 wks), visit 2 (6-8 wks), visit 3 (12 wks), visit 4 (6 mo), visit 5 ( $\geq 1$ yr). The number of patients $(\mathrm{N})$ with follow up data is listed below visit number

$\geq 30 \%$ change in NRS, SF-36, and ODI was also evaluated, in addition to corresponding point reductions roughly corresponding to historically reported MCIDs (Supplementary Figures 1, 2, and 3) [32-35]. Baseline NRS was also stratified into $>5(n=15)$ and $\leq 5(n=18)$ and was incorporated into analyses depicting $\geq 50 \%$ change for each outcome (Fig. 2). A series of sensitivity analyses were also performed to rule out the potential impact of missing data patterns as an alternative explanation for study conclusions.

\section{Results}

Medical records for 33 patients that were treated between April 2010 and April 2015 were reviewed which included 14 females and 19 males. Missing data patterns were consistent across patient sex. The average patient age was 45 (Table 2). Patients had a variety of pathologies related to disc disruption including: broad based disc bulges with annular tears, and small contained disc protrusions. Patients included in the analyses underwent a single treatment of intradiscal injection at $1(n=8), 2$ $(n=16)$, or $3(n=9)$ levels (Table 2$)$.

\section{Overall subjective scoring} NRS

The mean NRS $( \pm$ S.E.M) pre-injection was $5.2( \pm 0.4)$. The percentage of patients with at least $50 \%$ improvement in NRS scores at each post-injection time point (95\% confidence intervals) was $13.8 \%$ (1.2-26.3\%), 45.8\%
(25.9-65.8\%), 41.1\% (17.8-64.6\%), 23.5\% (3.4-43.7\%), and $38.9 \%(16.4-61.4 \%)$ at pre-injection, and at 2, 6-8, 12,24 , and $\geq 52$ weeks post-treatment, respectively (Fig. 1a). The proportion of patients with at least $30 \%$ improvement in NRS scores at each post-injection time point was also examined along with estimated MCID and is reported in Supplementary Figure 1.

\section{SF-36}

The mean SF-36 score $( \pm$ S.E.M) pre-injection was 53.4 $( \pm 2.6)$. The percentage of patients with at least $50 \%$ improvement in SF-36 scores at each post-injection time point (95\% confidence intervals) was $4.3 \%(0.0-12.7 \%)$, $28.6 \% \quad(4.9-52.2 \%), \quad 30.0 \% \quad(1.6-58.4 \%), \quad 22.2 \% \quad(0.0-$ $49.4 \%)$, and $30.8 \%(5.7-55.9 \%)$ at pre-injection, and at 2 , $6-8,12,24$, and $\geq 52$ weeks post-treatment, respectively (Fig. 1b). The proportion of patients with at least 30\% improvement in SF-36 scores at each post-injection time point was also examined along with estimated MCID and is reported in Supplementary Figure 2.

\section{$O D I$}

The mean ODI $( \pm$ S.E.M) pre-injection was $36.7( \pm 2.6)$. The percentage of patients with at least $50 \%$ improvement in ODI scores at each post-injection time point (95\% confidence intervals) was $4.2 \%(0.05-12.2 \%), 26.7 \%$ (4.3-49.0\%), 36.4\% (7.9-64.8\%), 55.6\% (23.1-88.0\%), and $30.8 \%(5.7-55.9 \%)$ at pre-injection, and at $2,6-8,12,24$, and $\geq 52$ weeks post-treatment, respectively (Fig. 1c). 

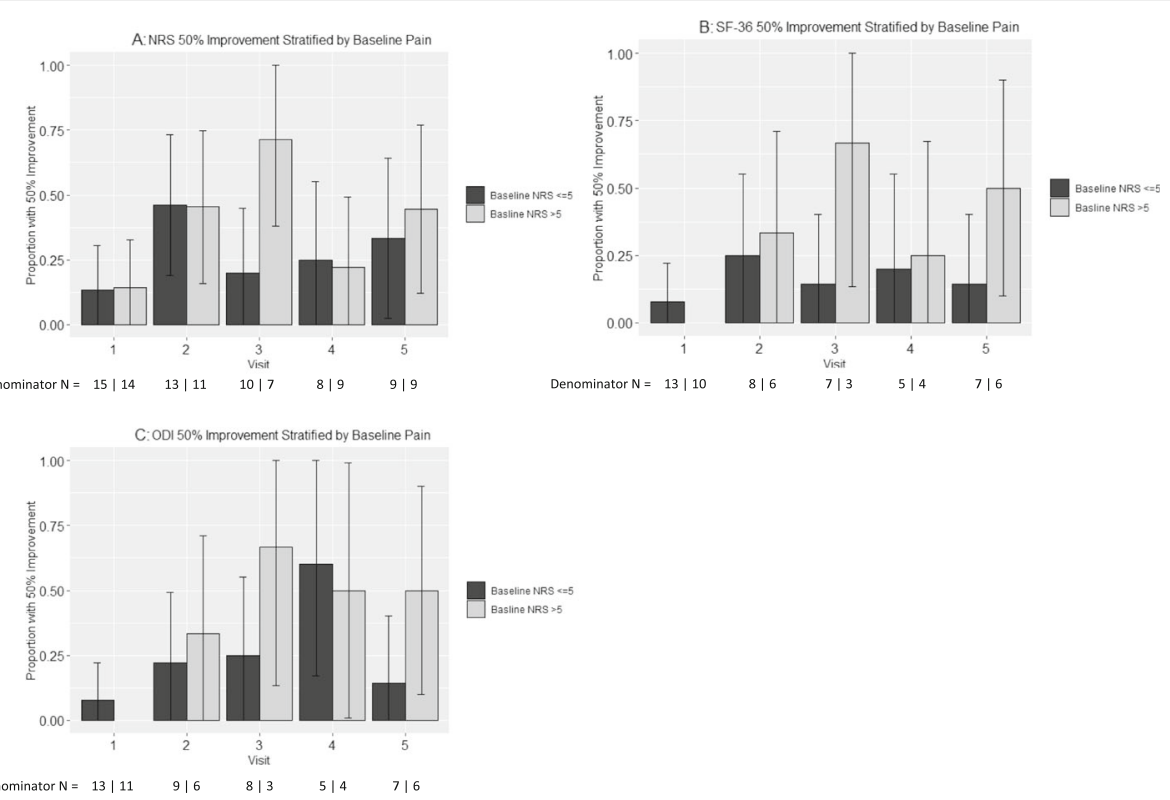

Fig. 2 Proportion of patients with Numeric Rating Scale (NRS; a), Short Form-36 Health Survey (SF-36; b) and Oswestry Low Back Pain Disability Index (ODI; c) score improvements of at least 50\%, and the number of patients included at each time point. Patients were subdivided into those with a pre-injection NRS $\leq 5$ and those with a pre-injection NRS $>5$. Values are proportions and $95 \%$ confidence intervals. Visits are defined as follows: visit 1 ( 2 wks), visit 2 (6-8 wks), visit 3 (12 wks), visit 4 (6 mo), visit 5 ( $\geq 1 \mathrm{yr})$. The number of patients ( $\mathrm{N}$ ) with follow up data is listed below visit number

The proportion of patients with at least $30 \%$ improvement in ODI scores at each post-injection time point was also examined along with estimated MCID and is reported in Supplementary Figure 3.

\section{Stratified subjective scoring (baseline NRS $\leq 5$ vs baseline NRS > 5)}

In secondary analyses, improvement scores were examined stratified by low baseline pain ( NRS $\leq 5, n=18)$ vs. high baseline pain (NRS $>5, n=15$ ).

\section{NRS}

The percentage of patients with an initial NRS $\leq 5$ at baseline, values were 13.3, 46.2, 20.0, 25.0, and 33.3\% at

Table 2 Baseline patient characteristics and patient reported outcome scores

\begin{tabular}{ll}
\hline & Mean or N \\
\hline Total \# Patients & 33 \\
Age & 45 (range 32-72) \\
Gender (Female/Male) & $14 / 19$ \\
1 Level Injection & 8 \\
2 Level Injection & 16 \\
3 Level Injection & 9 \\
Baseline NRS & $5.2 \pm 0.4$ \\
Baseline SF-36 & $53.4 \pm 2.6$ \\
Baseline ODI & $36.7 \pm 2.6$ \\
\hline
\end{tabular}

pre-injection, and at $2,6-8,12,24$, and $\geq 52$ weeks posttreatment, respectively (Fig. 2a). Among those with an initial NRS > 5 with at least 50\% improvement in NRS scores at each post-injection time point was $14.3,45.5$, $71.4,22.2$, and $44.4 \%$ at pre-injection, and at $2,6-8,12$, 24 , and $\geq 52$ weeks post-treatment, respectively.

\section{SF-36}

Improvement in SF-36 reported scores among those with an initial $\mathrm{NRS} \leq 5$, values were $7.8,25.0,14.3,20.0$, and $14.3 \%$, across follow-up visits at pre-injection, and at $2,6-8,12,24$, and $\geq 52$ weeks post-treatment. For those with an initial NRS $>5$ was $0.0,33.3,66.7,25.0$, and $50.0 \%$ at pre-injection, and at 2, 6-8, 12, 24, and $\geq 52$ weeks post-treatment, respectively (Fig. 2b).

ODI

Improvement in ODI among those with an initial NRS $\leq$ 5 , values were $7.7,22.2,25.0,60.0$, and $14.3 \%$ across follow-up visits at pre-injection, and at 2, 6-8, 12, 24, and $\geq 52$ weeks post-treatment. For those with an initial NRS $>5$ was $0.0,33.3,66.7,50.0$, and $50.0 \%$ at preinjection, and at $2,6-8,12,24$, and $\geq 52$ weeks posttreatment, respectively (Fig. 2c).

\section{Discussion}

An effective, pain-reducing, or possibly restorative treatment for chronic discogenic LBP would mark a major advancement in U.S. health care, since symptomatic 
management has historically been inadequate in relieving pain long-term. This 33-patient retrospective pilot study was performed to evaluate the safety and apparent effectiveness of non-surgical intradiscal injections of cBMA as a potential therapy for LBP. Based on commonly used patient reported validated outcome measures (NRS, SF-36, ODI), the major finding herein is that intradiscal injections of cBMA have the potential to reduce pain with a concomitant increase in overall patient health and function. Additionally, patients with higher baseline pain improved the most, which seems logical given that these patients had the greatest room for improvement.

This retrospective analysis has obvious limitations such as the lack of a control group, possible regression to mean, and incomplete patient data at certain time points. Thus, the interpretation of these results should be considered with prudence. Injections of concentrated Bone Marrow Aspirate were offered as a treatment option for qualified patients based upon clinical evaluation, the refractory and somewhat degenerative nature of their condition, and the relative absence of effective conservative rehabilitation strategies to avoid surgical intervention. Sub-stratification of internal disc disruption correlating to subjective response was not performed because multiple levels were treated, each with their own respective pathology. The presentation of this retrospective data was designed to report the clinical outcomes of a single physician, nonetheless it is useful to consider the similarities between these results and other larger prospective studies where CBMA and/or PRP were used to treat LBP [23-27]. For example, Tuakli-Wosurno et al. [24] observed a $35 \%$ reduction in pain from baseline to 8 weeks postinjection after injecting PRP intradiscally (NRS; 4.74 to 3.09 ); In the current study we observed a $40 \%$ reduction in pain during the same period in all patients (NRS; 5.2 to 3.1), and a $52 \%$ reduction in pain within the patients that had an initial NRS > 5 (NRS; 7.1 to 3.4). Similarly, Pettine et al. [23] reported a $63 \%$ reduction in pain in all subjects from baseline to 3 months post-injection with BMC (VAS; 79.3 to 29.2); In the current study we observed a $38 \%$ reduction in patient reported pain from baseline to 3 months post-injection (NRS; 5.2 to 3.2 ), and $62 \%$ reduction in pain within the patients that had an initial NRS score $>5$ (NRS; 7.1 to 2.7). The pain reductions within our dataset were consistent in direction but varied in magnitude at later time points. Patients with baseline NRS $>5$ had 32 and 38\% reductions at 6 months and 1 year, respectively, whereas Pettine et al. observed 67 and 58\% reductions at 6 months and 1 year, respectively [23]. Collectively, the similarities in therapeutic effectiveness are highly encouraging given that they occurred in the face of several differences among the studies (e.g., different physicians treating patients, diverse methods for product isolation, dissimilar patient populations, etc.), which could have caused widely variable results.
An interesting similarity to note is the apparent decrease in clinical effect around the 1-year mark for both this study and Pettine et al. [23]. Since each study statistically analyzed patients who only received a single cBMA injection, it is impossible to comment on whether an additional injection prior to the 1-year mark may provide a "rescue" effect. Although observational, two patients in the Pettine et al. study did choose to receive an additional injection between 6 and 12 months and both experienced additional pain relief. This topic has been fairly examined in the PRP literature, with a few high-level studies reporting that three PRP injections outperform the clinical benefit of a single PRP injection $[36,37]$. However, there are obvious limitations to the interpretation of each study (i.e. different follow-up time points, different disease states, etc.) and their relevance to cBMA injections for discogenic pain. For now, it seems prudent to focus on the biological responses and clinical outcomes from a single injection protocol. Future cBMA data is thus warranted to deliver a better answer to this question. Meanwhile, the reporting of $50 \%$ patient improvement proportions (Fig. 1), stratification of initial patient pain and function status (Fig. 2), and $30 \%$ patient improvement proportions along with MCID estimates (Supplementary Figures 1,2 and 3) from this study may help to improve potential patient benefits and expectations for these cellular therapies. The Supplementary Data reporting $\geq 30 \%$ improvement displays even stronger evidence for reduction in patient reported pain scoring for NRS, SF-36, and ODI.

There are several potential mechanisms that may explain these observed clinical improvements. An obvious contender would be that the addition of anabolic growth factors and regenerative stem cells to the degenerate IVD could offset its catabolic environment. Increased levels of growth factors and cytokines within PRP have been shown to improve AF and NP cell proliferation, increase glycosaminoglycan content and collagen synthesis, and stimulate gene expression for extracellular matrix proteins critical for IVD function [38, 39], even in the context of an inflammatory environment [40]. Similarly, cBMA also contains increased levels of growth factors and cytokines, yet additionally contains high levels of interleukin- 1 receptor antagonist protein (IL-1ra) [20] which effectively inhibits IL-1-mediated matrix degradation [41]. It is also well known that bone marrow contains a plethora of cells that can contribute to regeneration, both directly and through paracrine effects. Mesenchymal stem cells have received the greatest amount of attention (for reviews see $[9,42]$ ) possibly because they possess the potential for differentiation into IVD cells [43, 44]. However, their ability to increase proteoglycan content, secrete factors to reduce inflammation $[45,46]$, persist in the nutrient deprived IVD [47], and presence in the degenerate IVD [48] further supports the 
speculation that they are well-suited for treating LBP. When considering the large number of growth factors, cytokines, and regenerative cells present within cBMA it may be most appropriate to consider the way these components interact to regenerate the IVD. Further elucidation of the mechanisms involved are of great interest and important in understanding the utility of autologous therapies for LBP.

\section{Conclusions}

The development of therapies such as autologous cBMA to treat the IVD and discogenic pain would be advantageous to treat the large number of individuals affected by this pathology. Additional studies are necessary to identify which subset of patients with discogenic LBP are most likely to experience the highest and most consistent benefits from this minimally invasive autologous therapy, and how effective this therapy is when compared to control therapies. In the future we hope to report these results, however this retrospective analysis supports the contention that autologous based therapies, including cBMA, are a logical strategy to alleviate discogenic pain and restore patient function. The use of autologous cBMA or other autologous growth factors represents a paradigm shift not just aimed at mitigating symptoms, but with the goal of providing a restorative therapy which provides long-term benefits of reduced pain and improved disc health and function.

\section{Supplementary information}

Supplementary information accompanies this paper at https://doi.org/10. 1186/s12891-020-3126-7.

Additional file 1: Figure S1. (A) Proportion and 95\% confidence intervals of patients with Numeric Rating Scale (NRS) score improvements of at least 30\%. Proportions at each post-injection time point were 17.2, 54.2, 52.9, 41.2, and 44.4\%, respectively. (B) Estimated NRS MCID of 2 points for patients reporting at least $30 \%$ improvement in pain scores. Proportions at each post-injection time point were 17.2, 45.8, 47.1, 35.3, and $38.9 \%$, respectively. Visits are defined as follows: visit 1 (2 wks), visit 2 $(6-8 \mathrm{wks})$, visit $3(12 \mathrm{wks})$, visit 4 (6 mo), visit 5 ( $\geq 1 \mathrm{yr})$. The number of patients $(\mathrm{N})$ with follow up data is listed below visit number. Figure S2. (A) Proportion and 95\% confidence intervals of patients with Short Form-36 Health Survey (SF-36) score improvements of at least 30\%. Proportions at each post-injection time point were $4.4,35.7,30.0,44.4$, and $30.8 \%$, respectively. (B) Estimated SF-36 MCID of 18 points for patients reporting at least 30\% improvement in pain scores. Proportions at each post-injection time point were $4.4,35.7,30.0,44.4$, and $38.5 \%$, respectively. Visits are defined as follows: visit 1 (2 wks), visit 2 (6-8 wks), visit 3 (12 wks), visit 4 (6 $\mathrm{mo})$, visit $5(\geq 1 \mathrm{yr})$. The number of patients $(\mathrm{N})$ with follow up data is listed below visit number. Figure S3. (A) Proportion and 95\% confidence intervals of patients with Oswestry Low Back Pain Disability Index (ODI) score improvements of at least $30 \%$. Proportions at each post-injection time point were $8.3,46.7,45.5,66.7$, and $38.5 \%$, respectively. (B) Estimated ODI MCID of 12 points for patients reporting at least 30\% improvement in pain scores. Proportions at each post-injection time point were 8.3, $26.7,45.5,77.8$, and $30.8 \%$, respectively. Visits are defined as follows: visit 1 (2 wks), visit 2 (6-8 wks), visit 3 (12 wks), visit 4 (6 mo), visit 5 ( $\geq 1 \mathrm{yr}$ ). The number of patients $(\mathrm{N})$ with follow up data is listed below visit number

Additional file 2. Supplementary Dataset.

\section{Abbreviations}

AF: Annular fibrosis; BMAC: Bone marrow aspirate concentrate; BMC: Bone marrow concentrate; CBMA: concentrated bone marrow aspirate; IL1: Interleukin 1; IL-1ra: Interleukin 1 receptor antagonist protein; IVD: Intervertebral disc; LPB: Low back pain; MCID: Minimal Clinically Important Difference; MSC: Mesenchymal stem cell; NP: Nucleus pulposus; NRS: Numeric Rating Scale; ODI: Oswestry Low Back Pain Disability Index; PDGF: Platelet derived growth factor; PSIS: Posterior superior iliac spine; SF36: Short Form-36 Health Survey; TGF- $\beta$ : transforming growth factor beta; VAS: Visual Analog Scale; VEGF: Vascular endothelial growth factor

\section{Acknowledgements}

The authors would like to express their sincere gratitude to Amanda Busch for her invaluable assistance in the data collection for the preparation of this manuscript and Sarah Schmiege for her contributions with statistical analysis.

\section{Authors' contributions}

MW was primarily responsible for the conception and the design of this retrospective study: MW developed the procedural techniques, application, inclusion of patients, and data acquisition (with support from JMS). MW, JMS, $C R$, SKP, and BB were responsible for the analysis and the interpretation of the data. MW, JMS, CR, SKP, and BB all participated in the drafting and revising of the manuscript. All authors (MW, JMS, CR, SKP, and BB) read and approved the final manuscript.

\section{Funding}

There was no funding provided for this investigator-initiated retrospective analysis.

Availability of data and materials

The datasets supporting the conclusions of this article are included within the article and its additional files.

Ethics approval and consent to participate

The study was approved by the Honor Health Institutional Review Board (\#2011-041; June, 2012).

Consent for publication

All of the authors consent to publishing the paper in BMC Musculoskeletal Disorders Journal.

\section{Competing interests}

MW is a non-paid peer educator for Isto Biologics (formally Arteriocyte Medical Systems) who manufactures the device used in this study. BB \& SKP are employees of Isto Biologics.

\section{Author details}

${ }^{1}$ Southwest Spine and Sports, 9913 N. 95th St, Scottsdale, AZ 85258, USA ${ }^{2}$ University of Texas at San Antonio, San Antonio, TX, USA. ${ }^{3}$ Isto Biologics, Hopkinton, MA, USA.

Received: 15 July 2019 Accepted: 10 February 2020

Published online: 28 February 2020

\section{References}

1. Katz JN. Lumbar disc disorders and low-back pain: socioeconomic factors and consequences. J Bone Joint Surg Am. 2006;88:21-4.

2. Andersson GB. Epidemiological features of chronic low-back pain. Lancet. 1999;354:581-5.

3. DePalma MJ, Ketchum JM, Saullo T. What is the source of chronic low back pain and does age play a role? Pain Med. 2011;12:224-33.

4. Lyons G, Eisenstein SM, Sweet MB. Biochemical changes in intervertebral disc degeneration. Biochim Biophys Acta. 1981;673:443-53.

5. Marchand F, Ahmed AM. Investigation of the laminate structure of lumbar disc Anulus Fibrosus. Spine. 1990;15:402-10.

6. Chelberg MK, Banks GM, Geiger DF, Oegema TRJR. Identification of heterogeneous cell populations in normal human intervertebral disc. J Anat. 1995;186(Pt 1):43-53.

7. Urban JPG, Roberts S. Degeneration of the intervertebral disc. Arthritis Res Ther. 2003;5:120-30. 
8. Roberts S, Evans H, Trivedi J, Menage J. Histology and pathology of the human intervertebral disc. JBJS. 2006;88(Suppl 2):10-4.

9. Zeckser J, Wolff M, Tucker J, Goodwin J. Multipotent Mesenchymal stem cell treatment for Discogenic low Back pain and disc degeneration. Stem Cells Int. 2016;2016:3908389.

10. Long D, Liang S, Liu H, Wu X, Li Z, Wang H, Huang S, Zeng J. Mesenchymal stem cell in the intervertebral disc. In: Mesenchymal Stem Cells - Isolation, Characterization and Applications: IntechOpen; 2017. https://doi.org/10. 5772/intechopen.69296. Available from: https:/www.intechopen.com/ books/mesenchymal-stem-cells-isolation-characterization-and-applications/ mesenchymal-stem-cell-in-the-intervertebral-disc.

11. Filardo G, Kon E, Di Matteo B, Pelotti P, Di Martino A, Marcacci M. Plateletrich plasma for the treatment of patellar tendinopathy: clinical and imaging findings at medium-term follow-up. Int Orthop. 2013;37:1583-9.

12. Mishra A, Pavelko T. Treatment of chronic elbow tendinosis with buffered platelet-rich plasma. Am J Sports Med. 2006;34:1774-8.

13. Kon E, Mandelbaum B, Buda R, Filardo G, Delcogliano M, Timoncini A, Fornasari PM, Giannini S, Marcacci M. Platelet-rich plasma intra-articular injection versus hyaluronic acid viscosupplementation as treatments for cartilage pathology: from early degeneration to osteoarthritis. Arthroscopy. 2011:27:1490-501.

14. Gobbi A, Karnatzikos G, Scotti C, Mahajan V, Mazzucco L, Grigolo B. Onestep cartilage repair with bone marrow aspirate concentrated cells and collagen matrix in full-thickness knee cartilage lesions: results at 2-year follow-up. Cartilage. 2011;2:286-99.

15. Kim JD, Lee GW, Jung GH, Kim CK, Kim T, Park JH, Cha SS, You YB. Clinical outcome of autologous bone marrow aspirates concentrate (BMAC) injection in degenerative arthritis of the knee. Eur J Orthop Surg Traumatol. 2014;24:1505-11

16. Krych AJ, Nawabi DH, Farshad-Amacker NA, Jones KJ, Maak TG, Potter HG, Williams RJ. Bone marrow concentrate improves early cartilage phase maturation of a scaffold plug in the knee: a comparative magnetic resonance imaging analysis to platelet-rich plasma and control. Am J Sports Med. 2016:44:91-8.

17. Skoff HD. Revision rotator cuff reconstruction for large tears with retraction: a novel technique using autogenous tendon and autologous marrow. Am J Orthop. 2015:44:326-31.

18. Chahla J, Mannava S, Cinque ME, Geeslin AG, Codina D, LaPrade RF. Bone marrow aspirate concentrate harvesting and processing technique. Arthrosc Tech. 2017;6:e441-5.

19. Themistocleous GS, Chloros GD, Kyrantzoulis IM, Georgokostas IA, Themistocleous MS, Papagelopoulos PJ, Savvidou OD. Effectiveness of a single intra-articular bone marrow aspirate concentrate (BMAC) injection in patients with grade 3 and 4 knee osteoarthritis. Heliyon. 2018;4:e00871.

20. Cassano JM, Kennedy JG, Ross KA, Fraser EJ, Goodale MB, Fortier LA. Bone marrow concentrate and platelet-rich plasma differ in cell distribution and interleukin 1 receptor antagonist protein concentration. Knee Surg Sports Traumatol Arthrosc. 2016;26:333-42.

21. Imam MA, Mahmoud SSS, Holton J, Abouelmaati D, Elsherbini Y, Snow M. A systematic review of the concept and clinical applications of bone marrow aspirate concentrate in Orthopaedics. SICOT J. 2017;3:17.

22. Centeno CJ, Al-Sayegh H, Freeman MD, Smith J, Murrell WD, Bubnov R. A multi-center analysis of adverse events among two thousand, three hundred and seventy two adult patients undergoing adult autologous stem cell therapy for orthopaedic conditions. Int Orthop. 2016;40:1755-65.

23. Pettine KA, Murphy MB, Suziki RK, Sand TT. Percutaneous injection of autologous bone marrow concentrate cells significantly reduces lumbar discogenic pain through 12 months. Stem Cells. 2015;33:146-56.

24. Tuakli-Wosornu YA, Terry A, Boachie-Adjei K, Harrison JR, Gribbin CK, LaSalle EE, Nguyen JT, Solomon JL, Lutz GE. Lumbar Intradiskal platelet-rich plasma (PRP) injections: a prospective, double-blind, randomized controlled study. PM R. 2016:8:1-10.

25. Levi D, Horn S, Tyszko S, Levin J, Hecht-Leavitt C, Walko E. Intradiscal platelet-rich plasma injection for chronic Discogenic low Back pain: preliminary results from a prospective trial. Pain Med. 2016;17:1010-22.

26. Pettine KA, Suzuki R, Sand T, Murphy M. Treatment of discogenic back pain with autologous bone marrow concentrate injection with minimum two year follow-up. Int Orthop. 2016;40:135-40.

27. Pettine KA, Suzuki RK, Sand TT, Murphy MB. Autologous bone marrow concentrate intradiscal injection for the treatment of degenerative disc disease with three-year follow-up. Int Orthop. 2017;41:2097-103.
28. Nakagawa H, Morihara T, Fujiwara H, Kabuto Y, Sukenari T, Kida Y, Furukawa R, Arai Y, Matsuda Kl, Kawata M, Tanaka M, Kubo T. Effect of footprint preparation on tendon-to-bone healing: a histologic and biomechanical study in a rat rotator cuff repair model. Arthroscopy. 2017;33:1482-92.

29. Bogduk N. Practice guidelines for spinal diagnostic and treatment procedures. 2nd ed. San Francisco: ISIS; 2013. p. 420-58.

30. Marx RE, Stevens MR. Bone marrow aspiration and aspirate concentrate. In: Atlas of Oral and Extraoral bone harvesting. 1st ed. Hanover Park: Quintessence Publishing Co Inc; 2010. p. 141-9.

31. Oliver $K$, Awan T, Bayes M. Single- versus multiple-site harvesting techniques for bone marrow concentrate. Orthop J Sports Med. 2017:5:2325967117724398.

32. Davidson M, Keating JL, Eyres S. A low Back-specific version of the SF-36 physical functioning scale. Spine. 2004;29:586-94.

33. Lauridsen HH, Hartvigsen J, Manniche C, Korsholm L, Grunnet-Nilsson N. Responsiveness and minimal clinically important difference for pain and disability instruments in low back pain patients. BMC Musculoskelet Disord. 2006;:1-16.

34. Schwind J, Learman K, O'Halloran B, Showalter C, Cook C. Different minimally important clinical difference (MCID) scores lead to different clinical prediction rules for the Oswestry disability index for the same sample of patients. J Man Manip Ther. 2013;21:71-8.

35. Childs JD, Piva SR, Fritz JM. Responsiveness of the numeric pain rating scale in patients with low back pain. Spine (Phila Pa 1976). 2005:30:1331-4.

36. Gormeli G, Gormeli CA, Ataoglu B, Colak C, Aslanturk O, Ertem K. Multiple PRP injections are more effective than single injections and hyaluronic acid in knees with early osteoarthritis: a randomized, double-blind, placebocontrolled trial. Knee Surg Sports Traumatol Arthrosc. 2017:25:958-65.

37. Uslu Guvendi E, Askin A, Guvendi G, Kocyigit H. Comparison of efficiency between corticosteroid and platelet rich plasma injection therapies in patients with knee osteoarthritis. Arch Rheumatol. 2018;33:273-81.

38. Pirvu TN, Schroeder JE, Peroglio M, Verrier S, Kaplan L, Richards RG, Alini M, Grad S. Platelet-rich plasma induces annulus fibrosus cell proliferation and matrix production. Eur Spine J. 2014;23:745-53.

39. Akeda K, An HS, Pichika R, Attawia M, Thonar EJ, Lenz ME, Uchida A, Masuda K. Platelet-rich plasma (PRP) stimulates the extracellular matrix metabolism of porcine nucleus pulposus and anulus fibrosus cells cultured in alginate beads. Spine (Phila Pa 1976). 2006;31:959-66.

40. Kim HJ, Yeom JS, Koh YG, Yeo JE, Kang KT, Kang YM, Chang BS, Lee CK. Anti-inflammatory effect of platelet-rich plasma on nucleus pulposus cells with response of TNF-a and IL-1. J Orthop Res. 2014;32:551-6.

41. Le Maitre CL, Hoyland JA, Freemont AJ. Interleukin-1 receptor antagonist delivered directly and by gene therapy inhibits matrix degradation in the intact degenerate human intervertebral disc: an in situ zymographic and gene therapy study. Arthritis Res Ther. 2007;9:R83.

42. Richardson SM, Kalamegam G, Pushparaj PN, Matta C, Memic A Khademhosseini A, Mobasheri R, Poletti FL, Hoyland JA, Mobasheri A. Mesenchymal stem cells in regenerative medicine: focus on articular cartilage and intervertebral disc regeneration. Methods. 2016;99:69-80

43. Clarke LE, McConnell JC, Sherratt MJ, Derby B, Richardson SM, Hoyland JA. Growth differentiation factor 6 and transforming growth factor-beta differentially mediate mesenchymal stem cell differentiation, composition, and micromechanical properties of nucleus pulposus constructs. Arthritis Res Ther. 2014;16:R67.

44. Sakai D, Mochida J, Iwashina T, Watanabe T, Nakai T, Ando K, Hotta T. Differentiation of mesenchymal stem cells transplanted to a rabbit degenerative disc model: potential and limitations for stem cell therapy in disc regeneration. Spine. 2005;30:2379-87.

45. Bertolo A, Thiede T, Aebli N, Baur M, Ferguson SJ, Stoyanov JV. Human mesenchymal stem cell co-culture modulates the immunological properties of human intervertebral disc tissue fragments in vitro. Eur Spine J. 2011;20:592-603.

46. Murphy MB, Moncivais K, Caplan Al. Mesenchymal stem cells: environmentally responsive therapeutics for regenerative medicine. Exp Mol Med. 2013:45:e54.

47. Turner SA, Wright KT, Jones PN, Balain B, Roberts S. Temporal analyses of the response of intervertebral disc cells and Mesenchymal stem cells to nutrient deprivation. Stem Cells Int. 2016:2016:5415901.

48. Risbud MV, Guttapalli A, Tsai TT, Lee JY, Danielson KG, Vaccaro AR, Albert TJ, Gazit Z, Gazit D, Shapiro IM. Evidence for skeletal progenitor cells in the degenerate human intervertebral disc. Spine. 2007;32:2537-44.

\section{Publisher's Note}

Springer Nature remains neutral with regard to jurisdictional claims in published maps and institutional affiliations. 